\title{
GENE-ENZYME RELATIONSHIPS AT THE am LOCUS IN NEUROSPORA CRASSA
}

\author{
J. A. PATEMAN
}

Department of Genetics, University of Sheffield

and

J. R. S. FINCHAM

Department of Genetics, University of Leicester

\section{INTRODUCTION}

Received 14.x.57

THE amination deficient, am, locus in Neurospora crassa is of particular interest, since previous work (Fincham, 1954; Pateman, 1957), has shown that mutation at this locus specifically affects the formation of the enzyme glutamic dehydrogenase by the organism. A number of independently induced am mutants are available and a genetical and biochemical investigation of four of these is described in this paper.

\section{METHODS}

The origin of the experimental strains has been previously described (Pateman, 1957; Fincham and Pateman, 1957b) with the exception of $a m^{R}$ which is an amination deficient mutant kindly supplied by Dr J. L. Reissig. The mutants $a m^{32}, a m^{47}$ and $a m^{l}$ were induced by ultraviolet light, $a m^{29}$ by 20 -methylcholanthrene and $a m \Omega^{R}$ by $\beta$-propionolactone.

Media. For growth tests and for the keeping of stock cultures, either Fries No. 3 medium or the minimal medium " $\mathrm{N}$ " of Vogel and Bonner (1956) was used, with suitable supplements where necessary. Mycelium for enzyme extraction was grown on Fries No. 3 or on N-free buffered medium (Fincham, 1954) supplemented with $0.01 M$ ammonium tartrate and other supplements where necessary. All crosses between strains were made on medium favouring sexual reproduction. (Westergaard and Mitchell, 1947).

Enzyme extraction. Mycelial pads were grown in $50 \mathrm{ml}$. lots of medium in 250 or $35^{\circ} \mathrm{ml}$. conical flasks or in $150 \mathrm{ml}$. of medium in penicillin flasks for $4^{8} \mathrm{hr}$. at $25^{\circ} \mathrm{C}$. from a heavy inoculum of conidia. The cultures were not agitated during growth. The pads were thoroughly washed with distilled water, blotted to remove excess moisture, ground in $3-5$ times their weight of $0.05 M p \mathrm{H} 8.0$ or $p \mathrm{H} 7.4$ phosphate buffer $\left(\mathrm{KH}_{2} \mathrm{PO}_{4}-\mathrm{Na}_{2} \mathrm{HPO}_{4}\right)$ in a chilled mortar with powdered glass, and filtered by suction through a layer of kieselguhr after the volume of buffer had been made up to 20-30 times the weight of mycelium. The filtrates were almost clear and were used without dialysis. Protein contents of extracts were determined by the quantitative biuret procedure described elsewhere (Fincham, I954).

Glutamic dehydrogenase assay. Glutamic dehydrogenase activities were determined in the following system : I $\cdot 0 \mathrm{ml}$. extract ; $1 \cdot 6 \mathrm{ml}$. $\mathrm{O} \cdot \mathrm{I} M$ phosphate buffer, $p \mathrm{H} 8 \cdot 0$ or $p \mathrm{H} 7.4 ; 0 . \mathrm{I} \mathrm{ml.} \mathrm{o} \cdot \mathrm{I}$ or $0.2 \mathrm{M}$ sodium $\alpha$-ketoglutarate ; o.1 ml. o.1 $\mathcal{N}\left(\mathrm{NH}_{4}\right)_{2} \mathrm{SO}_{4}$; $0.2 \mathrm{ml}$. reduced triphosphopyridine nucleotide (TPNH) solution to give an optical density at $340 \mathrm{~m} \mu$ of $0 \cdot 3-0 \cdot 4$. The rate of oxidation of TPNH was followed by measuring the rate of decrease of optical density at $340 \mathrm{~m} \mu$ in a Unicam SP. 500 spectrophotometer at room temperature $\left(18-2 \mathrm{I}^{\circ} \mathrm{C}\right.$.). The reaction was usually 
started by the addition of the TPNH but, since many extracts oxidise, or otherwise destroy TPNH, at a very low rate independently of the glutamic dehydrogenase reaction, very low activities were measured as the difference between the rate before and after the addition of the ammonium salt. Specific activities are expressed as change in optical density $\times \mathrm{IO}^{3} / \mathrm{min} . / \mathrm{mg}$. protein. Specific activities obtained were considerably greater than those reported in an earlier paper (Fincham, 1954). This difference is probably due to the more nearly optimal conditions, particularly in respect of $p \mathrm{H}$ and $\alpha$-ketoglutarate concentration (Fincham, 1957), used for enzyme assay in the present work.

\section{WILD TYPES AND PSEUDOWILDS FROM $a m \times a m$ CROSSES}

(i) Crosses between am strains

It has been shown previously (Pateman, 1957) that the three independently induced mutations which resulted in the strains $\mathrm{am}^{29}$, $a m^{32}$ and $a m^{47}$ either occurred at the same locus or at very closely linked loci. A further series of crosses between the four strains $\mathrm{am}^{29}$, $a m^{32}, a m^{47}$ and $a m^{R}$ were set up comprising all six possible combinations of parents taken two at a time. In addition, crosses between identical am parents were up as controls. Ascospores from these crosses were spread on Fries No. 3 medium plus I 5 per cent. agar, I 5 per cent. sorbose, 0.75 per cent. sucrose and $0.02 M$ glycine contained in Petri dishes. The glycine strongly inhibits the growth of am ascospores with relatively little effect on wild-type spores (Pateman, 1957). The sorbose induces a colonial growth habit in any wild types which develop and facilitates isolation. The plates were kept at $60^{\circ} \mathrm{C}$. for 35 minutes immediately after spreading and then incubated at $25^{\circ} \mathrm{C}$. The total number of live ascospores was estimated by counting the germinated ascospores in twenty sample fields of each plate with a binocular dissecting microscope, after two days' incubation. The plates were examined daily and any colonies that developed were isolated and cultured; in two cases the number of colonies was large and a representative sample only was transferred. The results of these plating experiments are given in Table I.

The crosses between different $a m$ strains fell into two groups, which were distinct with respect to the frequency, time of development and appearance of the apparent wild-type colonies.

The apparent wild types from the $a^{29} \times a m^{32}$ and $a m^{32} \times a m^{47}$ crosses began to form visible colonies after 5-7 days' incubation and the colonies showed great variation in the rate and compactness of growth. When tested for growth on minimal medium, the apparent wild-type isolates in this group usually gave growth intermediate between that typical for $a m$ and $\mathrm{am}^{+}$strains. In fact, all these apparent wild types which were tested proved to be pseudowilds (see next section).

The apparent wild types from the other $a m \times a m$ crosses began to form visible colonies after 3-4 days' incubation; these colonies were uniform in appearance with a more vigorous, compact growth than 
the great majority of the pseudowild colonies. When tested for growth on minimal medium, wild-type isolates from these crosses showed growth fully comparable to that of stock wild types. When tested they all proved to be true wild types (see next section).

The frequency of true wild types from crosses between different am strains is very low and in the absence of suitable markers it is uncertain whether they are due to crossing-over or some such phenomenon as "gene conversion", or perhaps a combination of both. However, it is clear that the wild types are not due to normal back-mutation or contamination, since no wild types were recovered from the control crosses between identical am strains.

TABLE I

Frequency ond nature of wild types from am $\times$ am crosses

\begin{tabular}{|c|c|c|c|c|c|}
\hline Cross & $\begin{array}{l}\text { Total no. } \\
\text { ascospores }\end{array}$ & $\begin{array}{l}\text { Total no. } \\
\text { apparent } \\
\text { wild types }\end{array}$ & $\begin{array}{l}\text { Frequency } \\
\text { (per cent.) }\end{array}$ & Pseudowild & $\begin{array}{l}\text { True wild } \\
\text { types }\end{array}$ \\
\hline$a m^{29} \times a m^{29}$ & $5^{8} 5,000$ & 0 & $\ldots$ & 0 & 0 \\
\hline$a m^{32} \times a m^{32}$ & 629,000 & 0 & $\ldots$ & 0 & 0 \\
\hline$a m^{47} \times a m^{47}$ & 464,000 & o & $\ldots$ & o & o \\
\hline$a m R \times a m R$ & 5 I 2,000 & o & $\ldots$ & 0 & 0 \\
\hline$a m^{29} \times a m^{32}$ & 184,000 & $4^{26}$ & $c a .0 .23$ & $19 *$ & o \\
\hline$a m^{29} \times a m^{47}$ & $65 \mathrm{I}, 000$ & 14 & $c a .0 .002$ & 0 & 14 \\
\hline$a m^{29} \times a m R$ & $1,377,000$ & 3 & $c a .0 \cdot 0002$ & o & 3 \\
\hline$a m^{32} \times a m^{47}$ & I 56,000 & 87 & $c a .0 .06$ & $16 *$ & 0 \\
\hline$a m^{32} \times a m R$ & $\mathrm{I}, 293,000$ & Io & $c a .0 \cdot 0008$ & 0 & I0 \\
\hline$a m^{47} \times a m^{R}$ & 715,000 & 0 & $<0.0002$ & o & 0 \\
\hline
\end{tabular}

* Only a sample of the apparent wild types was transferred from the $a m^{29} \times a m^{32}$ and $a m^{32} \times a m^{47}$ crosses.

\section{(ii) Nature of apparent wild types from crosses between am strains}

All the apparent wild types isolated were backcrossed to one of the same parental am strains that had been used in the cross from which they were derived. Samples of ascospores from each of these backcrosses were plated on the selective medium described above. The germinated ascospores were examined after incubation for 2-3 days at $25^{\circ} \mathrm{C}$.

In those backcrosses which gave an approximate I : I segregation of wild-type and am ascospores, the wild-type parent was classified as a "true" wild type (see table I) since its behaviour was similar to that of the stock wild types which carried the $a m^{+}$allele. Crosses between wild types isolated from these backcrosses and am strains also gave I : I segregation for $\mathrm{am}$ and $\mathrm{am}^{+}$ascospores.

In backcrosses of apparent wild types derived from $a m^{29} \times a m^{32}$ and $\mathrm{am}^{32} \times \mathrm{am}^{47}$ there were no $\mathrm{am}^{+}$ascospores in the progeny, but after about 5 days, colonies developed, similar in frequency and every other respect to those from the original $a^{29} \times a m^{32}$ and $a^{32} \times a m^{47}$ crosses. Since these apparent wild types did not carry the $\mathrm{am}^{+}$allele 
and were intermediate in growth between $a m$ and $a m^{+}$strains on minimal and selective media, it seemed most probable that they were pseudowilds (Pittenger, 1954).

It is known that pseudowilds owe their origin to the presence of a disomic nucleus in an ascospore; if the homologous chromosomes carry mutant alleles which are complementary then the resultant strain may show a semi-wild phenotype. With consequent growth and nuclear division in the pseudowild, the disomic nuclei usually break down and a heterocaryon with two types of haploid nuclei, each type carrying a different mutant allele, is formed. In order to confirm the supposition that the apparent wild types were in fact pseudowilds, it was necessary to show that they originated as single ascospores and that am strains of two different types could be derived from them.

This was done as follows. From $a m^{29} \times a m^{32}$ and $a m^{32} \times a m^{47}$ crosses, a number of single ascospores which showed rather strong initial growth in the selective medium, were isolated. When tested for growth on minimal and supplemented medium, cultures from these single ascospore isolates gave intermediate growth (see table 4). All the single ascospore cultures behaved like pseudowilds in backcrosses to am strains.

Representative strains, I28I from $a m^{29} \times a m^{32}$ and 1214 from $a m^{32} \times a m^{47}$, were used in the following experiments. A number of single conidium isolates were made from sorbose plates streaked with conidial suspensions. All of these isolates were tested for growth on minimal medium and many behaved like typical am strains. A number of am isolates from I 28I were tested in pairs for heterocaryon formation by superimposing drops of thick conidial suspensions on glycine sorbose plates. These am isolates fell into two groups; combinations involving one strain from each group gave comparatively vigorous colonies, presumably by heterocaryon formation. All the colonies which originated from mixed conidial suspension were cultured and tested for growth on minimal medium. In a similar fashion complementary heterocaryons were formed between am isolates from I2 I4. The dry weights of mycelia from tests of the am isolates and complementary heterocaryons in growth flasks are given in table 4. It seems clear from this evidence that the intermediate wild types from $a m^{29} \times a m^{32}$ and $a m^{32} \times a m^{47}$ were pseudowilds which gave rise to complementary heterocaryons.

It did not appear to make any difference whether the heterocaryons originated from disomic nuclei or by vegetative hyphal fusion, though pseudowild formation provided an easy, effective method of overcoming incompatibility difficulties in heterocaryon formation. The possibility remains that the semi-wild phenotype of these heterocaryons was, in fact, due wholly or in part to the presence of some disomic or diploid nuclei. This question is not settled, but evidence presented in a later section suggests that the possibility is unlikely. It is of some 
interest that from no one type of cross were both true wild types and pseudowilds recovered. In those types of cross from which only true wild types were obtained, it is certain that no pseudowilds were formed, since all colonies were transferred and analysed. But it is possible that rare true wild types occurred in those crosses which produced large numbers of pseudowilds. The great majority of pseudowild and true wild-type colonies are distinct in appearance and time of development on the plates, but there is some overlap. Consequently, a rare, true wild type, which developed late, might not have been obviously different from surrounding pseudowild colonies and might not, by chance, have been included in the small sample of those transferred for testing. However, the experiments

TABLE 2

Enzyme activities of true wild types

\begin{tabular}{|c|c|c|c|c|c|c|c|c|}
\hline \multicolumn{3}{|l|}{ Strains } & \multicolumn{6}{|c|}{$\begin{array}{c}\text { Specific glutamic dehydrogenase } \\
\text { activities }\end{array}$} \\
\hline I4 wild types from $a m^{29} \times a m^{47}$ & - & . & $\begin{array}{l}410 \\
590 \\
710\end{array}$ & $\begin{array}{r}715 \\
1062\end{array}$ & $\begin{array}{l}505 \\
820\end{array}$ & $\begin{array}{l}600 \\
320^{*}\end{array}$ & $\begin{array}{l}55^{\circ} \\
35^{\circ}\end{array}$ & $\begin{array}{l}850 \\
420\end{array}$ \\
\hline 3 wild types from $a m^{29} \times a m R$. & - & . & $54^{\circ}$ & 470 & 535 & & & \\
\hline Io wild types from $a m^{32} \times a m R$. & - & . & $\begin{array}{l}665 \\
897\end{array}$ & $\begin{array}{l}977 \\
472\end{array}$ & $\begin{array}{l}825 \\
824\end{array}$ & $\begin{array}{l}650 \\
768\end{array}$ & 594 & 595 \\
\hline
\end{tabular}

* Assayed at $p \mathrm{H} 7 \cdot 4$.

described and other work on the recovery of true wild types from $a m^{29} \times a m^{32}$ and $a m^{32} \times a m^{47}$ crosses (Pateman, I956), suggests that if they occur, the frequency is at least no greater than that found for the other am intercrosses.

\section{ENZYME ACTIVITIES OF WILD TYPES AND PSEUDOWILDS}

(i) All the true wild types from $a m^{29} \times a m^{47}, a m^{29} \times a m^{R}$ and $a m^{32} \times a m^{R}$ were assayed for glutamic dehydrogenase activity. The results are given in table 2 . There was some variation between the specific enzyme activities of the different wild types, but there was no evidence to suggest that any of them was significantly different from the stock wild types carrying the $a m^{+}$allele.

(ii) A representative sample of pseudowilds from $a m^{29} \times a m^{32}$ and $a m^{32} \times a m^{47}$ were assayed for glutamic dehydrogenase activity. The results are given in table 3 . There was a good deal of variation in the enzyme activities of the pseudowilds, but this was probably due to uncontrollable factors such as varying nuclear ratio. In no case, so far, has the enzyme activity of a pseudowild extract approached that of the stock wild types. 
(iii) Table 4 shows the results of the enzyme assays of am isolates and reconstituted heterocaryons derived from I28I and I 2 I4, which are single ascospore pseudowilds from $a m^{29} \times a m^{32}$ and $a m^{32} \times a m^{47}$ respectively. These results illustrate the remarkable fact that certain am alleles, individually incapable of detectable glutamic dehydrogenase activity, are complementary in function in heterocaryons with the resultant production of enzyme. Moreover, it seems probable that this complementary action is between alleles in different nuclei in the heterocaryon.

TABLE 3

Growth on minimal medium and enzyme activities of pseudowilds

\begin{tabular}{|c|c|c|c|c|}
\hline \multirow[b]{2}{*}{ Strains } & & \multicolumn{2}{|c|}{ Dry weight of mycelium in $\mathrm{mg}$. } & \multirow{2}{*}{$\begin{array}{c}\text { Specific glutamic } \\
\text { dehydrogenase } \\
\text { activity }\end{array}$} \\
\hline & & Fries minimal & $\begin{array}{c}\text { Fries plus } \\
\mathrm{M} / 200 \\
\text { DL-alanine }\end{array}$ & \\
\hline I $34^{8} \mathrm{am}^{+}$. & • & 43 & $4^{2}$ & $325^{*}$ \\
\hline Pseudowilds from $a m^{29} \times a m^{32}$ & $\begin{array}{l}\text { I } 279 \\
\text { I } 280 \\
\text { I } 28 \text { I } \\
\text { I } 283 \\
\text { I } 284\end{array}$ & $\begin{array}{l}28 \\
28 \\
26 \\
29 \\
3 \mathrm{I}\end{array}$ & $\begin{array}{l}36 \\
31 \\
34 \\
43 \\
47\end{array}$ & $\begin{array}{l}49^{*} \\
77^{*} \\
40^{*} \\
57^{*} \\
97^{*}\end{array}$ \\
\hline Pseudowilds from $a m^{32} \times a m^{47}$ & $\begin{array}{l}\text { I } 2 \text { I } 4 \\
\text { I } 2 \text { I } 5 \\
\text { I } 2 \text { I } 6 \\
\text { I } 2 \text { I } 7\end{array}$ & $\begin{array}{r}3 \\
1 \\
9 \\
14\end{array}$ & $\begin{array}{l}33 \\
28 \\
30 \\
3^{\circ}\end{array}$ & $\begin{array}{r}60 \\
99 \\
67 \\
14\end{array}$ \\
\hline
\end{tabular}

Cultures for growth tests were grown from small conidial inocula for 72 hours at $25^{\circ} \mathrm{C}$. in $100 \mathrm{ml}$. flasks containing $15 \mathrm{ml}$. of medium.

$$
\text { * Assayed at } p \mathrm{H} 7 \cdot 4 \text {. }
$$

\section{PROPERTIES OF FORCED HETEROCARYONS}

In order to extend the information derived from study of pseudowild types, heterocaryons carrying various combinations of am alleles were synthesised. The different $a m$ strains were each crossed to two arginine-requiring mutants, 46004 (arg-I, linkage group I) and $\mathrm{B}_{3} \mathrm{I} 7$ (arg-Io, linkage group VII). These mutants were supplied by Dr Dorothy Newmeyer, who has established their linkage relationships (Newmeyer, 1957). Double mutants were isolated from the crosses, and heterocaryon formation was forced by inoculating mixtures of conidia from $\arg$-I and $\arg$-1o strains on to agar slants lacking arginine but supplemented with alanine. Only double mutants which formed heterocaryons readily with the original $\arg$-I and $\arg$-Io strains were used for heterocaryon formation; all were of mating type $a$.

Table 5 shows the results of enzyme assays on heterocaryons formed between strains which, individually, were incapable of forming detectable glutamic dehydrogenase. The combinations $a m^{32}+a m^{47}$ 
and $a m^{32}+a m^{29}$ were found to produce the enzyme though at considerably less than the usual wild-type level. All other types of heterocaryons formed no detectable glutamic dehydrogenase in repeated experiments ; the minimum activity which should have been detectable

\section{TABLE 4}

Growth on minimal medium and enzyme activities of am isolates and reconstituted heterocaryons from pseudorvilds

\begin{tabular}{|c|c|c|c|}
\hline \multirow[b]{2}{*}{ Strain } & \multicolumn{2}{|c|}{ Dry weight of mycelium in mg. } & \multirow{2}{*}{$\begin{array}{l}\text { Specific glutami } \\
\text { dehydrogenase } \\
\text { activity }\end{array}$} \\
\hline & Fries minimal & $\begin{array}{c}\text { Fries plus } \\
\mathrm{M} / 200 \\
\text { DL-alanine }\end{array}$ & \\
\hline Pseudowild I 28I from $a m^{29} \times a m^{32}$. & 26 & 34 & $4^{*} *$ \\
\hline 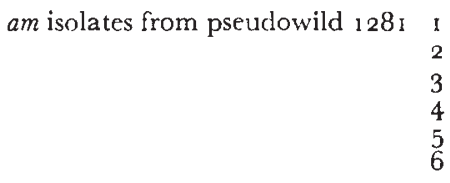 & $\begin{array}{l}0 \\
0 \\
0 \\
0 \\
0 \\
0\end{array}$ & $\begin{array}{l}29 \\
24 \\
23 \\
25 \\
24 \\
22\end{array}$ & $\begin{array}{l}\text { o }(<3) \\
\text { o }(<3) \\
\text { o }(<3) \\
\text { o }(<3) \\
\text { o }(<3) \\
\text { o }(<3)\end{array}$ \\
\hline $\begin{array}{cr}\text { Heterocaryons between } & \text { am } \\
\text { isolates from 1281 } & 1+4 \\
& 1+5 \\
& 2+3 \\
& 2+6\end{array}$ & $\begin{array}{l}26 \\
30 \\
29 \\
37\end{array}$ & $\begin{array}{l}33 \\
37 \\
37 \\
38\end{array}$ & $\begin{array}{l}80 \\
49 \\
96 \\
64\end{array}$ \\
\hline Pseudowild 1214 from $a m^{32} \times a m^{47}$. & 3 & 33 & 60 \\
\hline am isolates from pseudowild $1214 \begin{array}{r}1 \\
2 \\
\\
3 \\
4 \\
4\end{array}$ & $\begin{array}{l}0 \\
0 \\
0 \\
0\end{array}$ & $\begin{array}{l}30 \\
34 \\
33 \\
34\end{array}$ & $\begin{array}{l}\text { o }(<3) \\
0(<0 \cdot 6) \\
0(<2) \\
0(<1)\end{array}$ \\
\hline $\begin{array}{cr}\text { Heterocaryons between } & \text { am } \\
\text { isolates from 1214 } & 1+4 \\
& 2+3 \\
& 3+4\end{array}$ & $\begin{array}{r}10 \\
2 \\
9\end{array}$ & $\begin{array}{l}35 \\
33 \\
29\end{array}$ & $\begin{array}{l}75 \\
82 \\
84\end{array}$ \\
\hline $1346 \mathrm{am}^{+}$ & 35 & 33 & $55^{\circ}$ \\
\hline
\end{tabular}

* Assayed at $p \mathrm{H} 7 \cdot 4$.

in each experiment is indicated in table 5 and varied according to the protein content of the extract and the stability of the spectrophotometer at the time. These results were checked by growth experiments. The heterocaryons which produced glutamic dehydrogenase grew almost as well as the wild type on minimal medium, while those which formed no detectable glutamic dehydrogenase grew no better on minimal medium than did homocaryotic am strains (i.e. no weighable growth after 3 days at $25^{\circ} \mathrm{C}$.), although they grew at approximately wild-type rates on medium supplemented with DL-alanine at $\frac{1}{2} \mathrm{mg}$. per ml.

The allele $a m^{l}$, which is described in detail elsewhere (Fincham 
and Pateman, 1957 $\mathrm{b}$; Fincham, 1957) was isolated as an apparent back-mutant in an experiment in which conidia of an $\mathrm{am}^{\mathbf{4 7}}$ strain were irradiated with ultraviolet light. The available evidence (Fincham, I957) supports the hypothesis that $\mathrm{am}^{l}$ produces a glutamic dehydrogenase qualitatively different from that of the wild type. Extracts of $a m^{l}$ strains have low glutamic dehydrogenase activity which can be increased to a level approaching that of wild type by warming to $35^{\circ} \mathrm{C}$. or higher for a few minutes. This extra activity is slowly lost on returning the activated extract to room temperature, but can be regained on repeated warming. Treatment at $35-50^{\circ} \mathrm{C}$. does not affect the activity of wild-type extracts, nor does it induce

TABLE 5

Specific glutamic dehydrogenase activities from forced heterocaryons

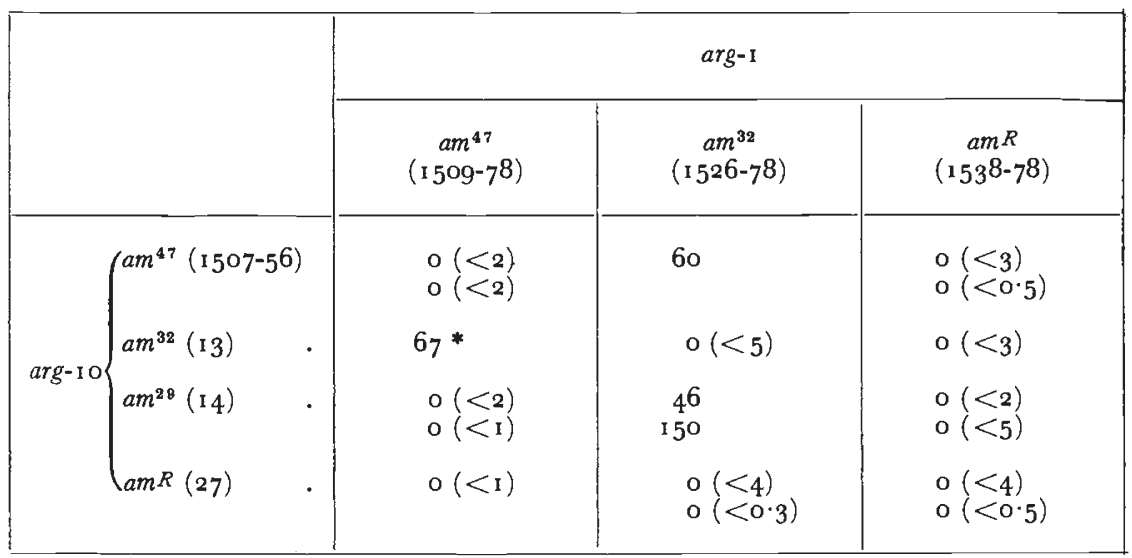

* Grown on Vogel and Bonner's medium " $\mathrm{N}$ " instead of the usual N-free buffered medium with ammonium tartrate.

The medium was supplemented with DL-alanine in all cases, either $\frac{1}{2} \mathrm{mg}$. or $\mathrm{I} \mathrm{mg} . / \mathrm{ml}$. Wild type typically gave specific activities of 500 or more grown under the same conditions.

activity in extracts of other am mutants lacking glutamic dehydrogenase. The factor by which the activity of $a m^{l}$ extract is increased by warming varies from experiment to experiment, but has always been at least six in the present series of experiments (table 6). The variation is thought to be due to differences in room temperature; the effect of warming has generally been less at higher room temperatures at which $\mathrm{am}^{l}$ enzyme is probably already partly activated or rapidly becomes so on mixing with substrates.

Results of experiments on heterocaryons carrying $a m^{l}$ in combination with other am alleles are shown in table 6. Extracts of heterocaryons of the type $a m^{l}+a m^{47}$ were qualitatively similar to $a m^{l}$ homocaryons, although their activity was generally rather less. Thus there is no indication of any complementary interaction between these two alleles. On the other hand, extracts of $a m^{l}+a m^{32}$ heterocaryons behaved as if they contained a mixture of wild-type and 
$a m^{l}$-type glutamic dehydrogenases (Fincham, 1957); their activity before heat treatment was very much greater than that of typical

TABLE 6

Enzyme activities of heterocaryons involving $a m^{1}$

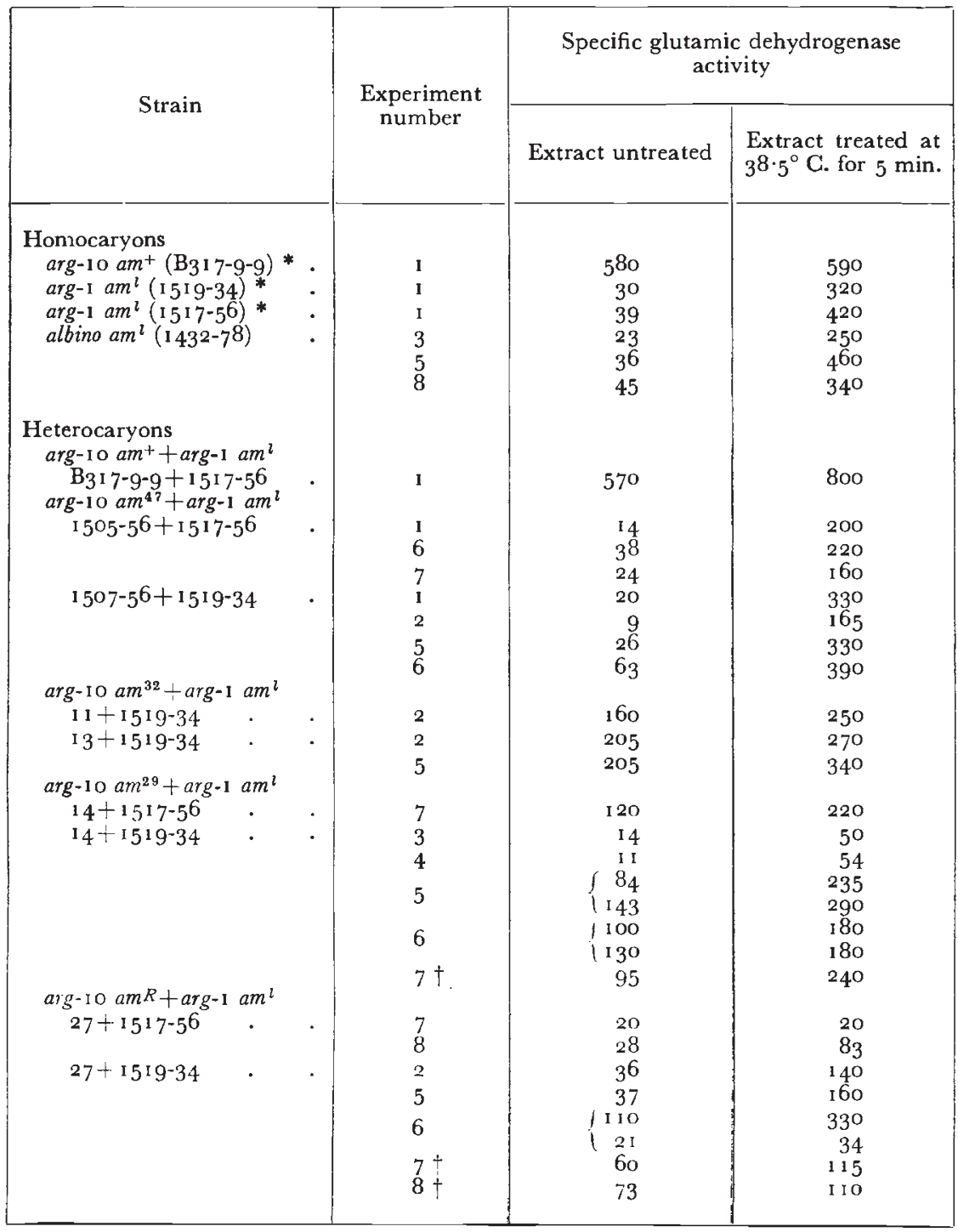

* Grown in medium supplemented with $\frac{1}{2} \mathrm{mg} . / \mathrm{ml}$. L-arginine hydrochloride.

$\dagger$ Heterocaryon made afresh from the original stocks for this experiment.

Values derived from the same experiment arc more closely comparable than those from different experiments, owing to differences in room temperature.

$a m^{l}$ extracts, but their response to heat was very much less. This result was not unexpected in view of the complementary interaction already shown between $a m^{47}$ and $a m^{32}$. More surprisingly, both $a m^{l}+a m^{29}$ and $a m^{l}+a m^{R}$ heterocaryons gave evidence of producing 
some enzyme resembling wild-type glutamic dehydrogenase in not responding to the temperature treatment. Extracts of heterocaryons of these two types sometimes had rather low activity before warming, but in no case was the stimulation of activity by warming as great as in extracts of $a m^{l}$ or $a m^{l}+a m^{47}$ cultures tested in the same experiment. The rather variable behaviour of these heterocaryons in replicated experiments is unexplained; it could perhaps be due to fluctuations in nuclear ratio. The data as a whole suggest that $a m^{l}$ is capable of interacting with either $a m^{32}, a m^{29}$ or $a m^{R}$ (but not with $a m^{47}$ ) to produce glutamic dehydrogenase of the normal type. There is a strong indication that the combination $a m^{l}+a m^{R}$ is less effective in this respect than $a m^{l}+a m^{32}$, with $a m^{l}+a m^{29}$ perhaps occupying an intermediate position. These provisional conclusions could be better tested by experiments in which nuclear ratios were controlled. It remains possible that some of the heterocaryons involving $a m^{l}$ may produce still further varieties of glutamic dehydrogenase, but the investigation of this possibility depends on the development of other methods for distinguishing types of enzyme.

\section{IS THE FORMATION OF DIPLOID NUCLEI THE BASIS OF COMPLEMENTARITY IN HETEROCARYONS?}

Perhaps the most surprising aspect of the complementarity shown by certain pairs of am alleles is the fact that it apparently operates with the different alleles in separate nuclei. The question arises whether the interaction is actually due to the occasional spontaneous fusion of nuclei such as is known to occur in Aspergillus nidulans (Pontecorvo et al., I 953). If diploid nuclei are responsible for the enzyme production in $a m^{x}+a m^{y}$ heterocaryons they must presumably be present in the mycelium in fair frequency to account for the levels of enzyme found, since a previous study (Fincham, I954) indicated that the level of glutamic dehydrogenase in heterocaryons was not far from being proportional to the percentage of $a m^{+}$nuclei. On the other hand, stable diploids have never been demonstrated in Neurospora, although it is known that they have been looked for without success by several investigators. Thus it seems probable that diploid nuclei, if they occur, are highly unstable and become readily reduced to haploids (perhaps by a process analogous to that by which a haploid heterocaryon arises from the presumably originally disomic nucleus of a pseudowild ascospore). Such diploidisation and subsequent reduction would be accompanied by free recombination of unlinked markers. To test this possibility a search was made for conidia, produced by a heterocaryon of type $\operatorname{arg-I} a^{47}+\operatorname{arg-Io} \mathrm{am}^{32}$, which had become homocaryotic for one or other arg mutant, while remaining heterocaryotic with respect to the am alleles. Conidia were spread on agar plates supplemented with $0.3 \mathrm{mg}$. of L-arginine hydrochloride per ml., a concentration of arginine which satisfies an arginine requirement without permitting optimal growth of am 
conidia. A total of IO2 well-grown sporelings were isolated. All except 3 were arginine-independent, and the three which required arginine all proved to be homocaryotic for $\mathrm{am}$. A further 75 sporelings which appeared to be growing relatively poorly on the original plate were isolated. Of these 73 required arginine, and all were homocaryotic for am; the other two were arginine-independent and behaved as if heterocaryotic for $a m^{32}+a m^{47}$. Thus there was no evidence for somatic recombination such as might be expected if unstable diploid nuclei were being formed. A further possibility is that diploid nuclei are formed which are effective in promoting enzyme formation but undergo no further division; such diploid nuclei could only be detected by cytological methods which have not yet been developed.

\section{DISCUSSION}

There are two main points of interest in the results described. First, the complementary action of certain pairs of am mutants in the production of glutamic dehydrogenase. Second, the genotypic status of the different am mutants and what information can be deduced from them concerning the organisation of that genetic material called the am locus, which apparently plays some specific role in the formation of glutamic dehydrogenase in Neurospora. These two points are of course inter-related, but for convenience will be discussed separately.

If, as seems probable, glutamic dehydrogenase synthesis may be promoted by the joint action of two kinds of separate nuclei, neither of which is capable of forming the enzyme by itself, a chromosomeenzyme relationship of unexpected complexity seems to be indicated. There is little doubt that Neurospora glutamic dehydrogenase is a single enzyme; as reported elsewhere (Fincham and Pateman, 1957a) neither mixtures of $a m^{32}$ and $a m^{47}$ extracts nor extracts of mixtures of $a m^{32}$ and $a m^{47}$ mycelium have been found to possess any activity, so these two mutants evidently do not produce different components of a complex enzyme system. Moreover, Neurospora glutamic dehydrogenase has recently been purified 40-fold (Fincham, unpublished) without great loss of total activity. The data could be interpreted as suggesting that glutamic dehydrogenase is formed in a series of distinct steps under the control of different parts of the am locus. In the positive heterocaryons one can imagine an intermediate in enzyme synthesis diffusing from one nucleus to another, if the enzyme is synthesised in the nucleus, or from one gene product in the cytoplasm to another, if it is synthesised in the cytoplasm. The difficulty with such a picture, apart from the current trend of biochemical opinion against multi-step enzyme synthesis (see, for example, Spiegelman, with added comment by Velick, I956), is that it provides no obvious reason why the genes concerned with the various steps should be tightly organised in a complex locus in the wild type. As an alternative we suggest that the whole am locus may, in the wild type, act as a 
unit in producing a single product (or plasmid, in Catcheside's (I956) terminology) which passes into the cytoplasm and becomes an essential part of the glutamic dehydrogenase-forming system. In the heterocaryons nuclear products with non-overlapping defects can be imagined as becoming organised in such a way, presumably through being built in to the same structure, that they can form a synthetic functional unit. A similar suggestion has been made by. Giles et al. (r957) for their parallel case involving adenylosuccinase formation. Such a hypothesis requires that nuclear products be incorporated at specific cytoplasmic sites with rather high efficiency, since it is otherwise difficult to explain how the complementation could work as well as it apparently does, even though the heterocaryons have not been found to approach the wild type in efficiency of enzyme production.

The present information on the physiological relationships of the am mutants can be represented by a linear diagram (fig. I). Mutations

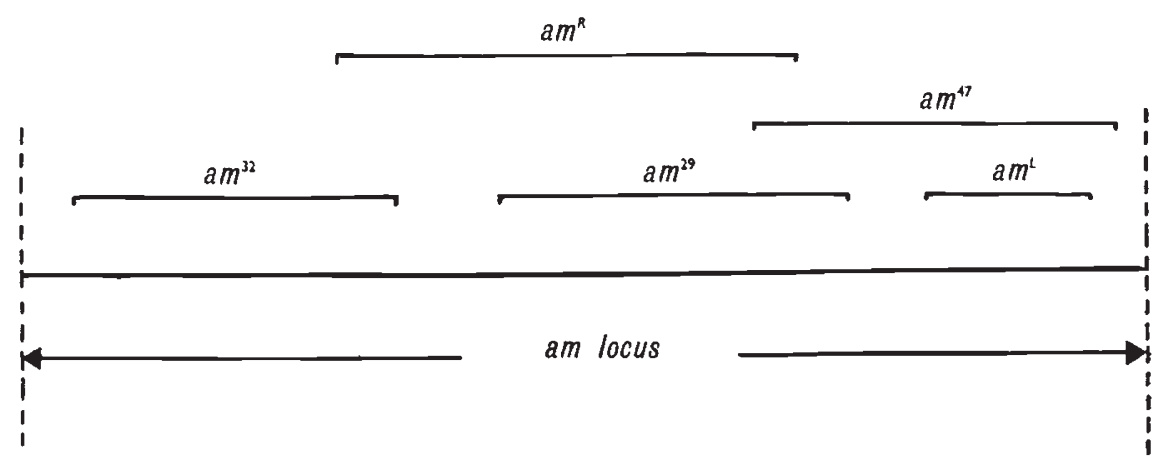

FIG. 1.-A diagrammatic representation of the physiological relationships between heteroalleles at the am locus.

which are mutually complementary may be envisaged as affecting non-overlapping regions of the complex locus, while mutations which are non-complementary may affect overlapping regions. Whether such a diagrammatic representation bears any relationship to the actual spatial organisation of the locus is doubtful.

Any tentative conclusions that might be made concerning the genetic status of the am mutants investigated, depend to a great extent on the mode of origin postulated for the wild types recovered from certain am intercrosses. In the absence of tetrad analysis with suitable markers, it is not possible to say whether these wild types arise as a result of orthodox crossing-over, gene conversion, or a combination of both. But it is probably significant that the wild-type frequencies from am intercrosses do not support any postulated linear arrangement of $a m$ " alleles". For example, in fig. 2 some of the wild-type frequencies from am intercrosses are represented in a linear diagram. This is one of the more plausible possible arrangements, yet there are two discrepancies with the rest of the data, (I) the frequency of wild types from $a m^{R} \times a m^{47}$ is less than 0.0002 per cent.; (2) there were no true wild types from $a m^{32} \times a m^{47}$. Furthermore, any attempt to 
incorporate the data on complementarity and wild-type recovery in a single linear scheme, raises a number of anomalies. Thus it seems unlikely that all of the recovered wild types can have been the result of orthodox crossing-over, at least a proportion and possibly all of them may have been due to gene conversion. This is in line with other work on yeast (Lindegren, I955; Winge, I955 ; Roman, I956), Neurospora (Mitchell, r955a, $b$; St Lawrence, I956 ; Giles, I95I) and possibly Aspergillus (Pritchard, I955). The fact that gene conversion must at least be taken into account makes it vastly more difficult to relate wild-type formation to the actual topography of the am locus. It has already been pointed out by St Lawrence and Bonner (1957) that the demonstration of gene conversion in organisms such as fungi, makes the interpretation of recombinant frequencies in terms of detailed spatial organisation of the genetic material a somewhat hazardous procedure. The elegant and refined analysis of the genetic material of a bacteriophage by Benzer (1957) may only be possible

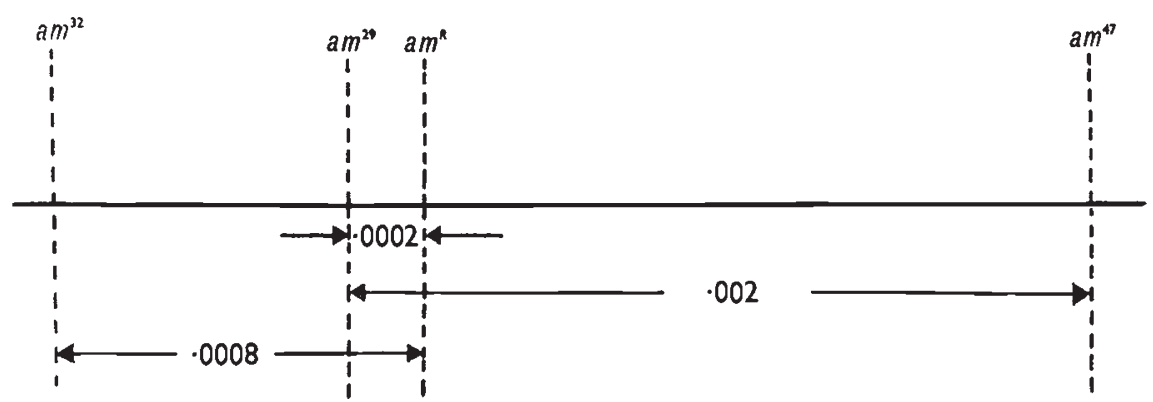

FIG. 2.-A linear diagram based on wild-type frequencies obtained from some of the am intercrosses.

with this type of organism and not applicable to more complex forms of life.

In order to overcome certain difficulties involved in the classical concept of the "gene", Benzer (I957) proposed the acceptance, in its place, of three fundamental genetic units, the " recon", the "muton" and the "cistron". The cistron is the functional unit and two apparently similar mutants which are non-complementary in a heterocaryon are considered to be located in the same cistron. Thus $a m^{32}$ would be in a different cistron to $a m^{29}$ and $a m^{47}$. The position of $a m^{R}$ is not so clear, since it is not complementary with $a m^{29}, a m^{32}$ or $a m^{47}$; it might be a deletion or defect which overlaps the other am sites, but this seems improbable since true wild types have been recovered from $a m^{29} \times a m^{R}$ and $a m^{32} \times a m^{R}$ crosses. The case of the am mutants raises questions of terminology not covered by Benzer's scheme. The entity which we have referred to in this paper as the am "locus" is clearly some kind of significant unit since $(a)$ it is concerned as a whole with the synthesis of a single specific enzyme, (b) its parts are extremely closely linked (and perhaps hardly separable by orthodox crossing-over), and $(c)$ it is not analysable into two or 
more discrete cistrons. One may ask whether the cistron is the significant functional unit in all cases and whether some other term may not be necessary to cover such cases as the present. Until more information is available on the generality of this kind of situation, we propose to continue to use the term "locus".

In view of these considerations and with the data at present available, it seems best to regard the different am mutants as carrying different "heteroalleles" (Roman, I956, and Beadle, I957) at the am locus. This terminology recognises the basic fact that the am mutants carry demonstrably different components of the am locus, between which some form of gene conversion (transmutation, Beadle, I957) can probably occur. Whether or not crossing-over can take place between these heteroalleles has yet to be satisfactorily demonstrated. The fact that certain pairs of heteroalleles are physiologically complementary provides a further criterion for distinguishing between them. It is obvious that more experimental work requires to be done with, if possible, a further group of independently induced am mutants, in order to extend our understanding of the gene-enzyme relationships at the am locus.

\section{SUMMARY}

(I) It has been shown that wild types can be recovered from crosses between different am mutants. From certain intercrosses only true wild types with a very low frequency were obtained, while from the other crosses only pseudowilds with a comparatively high frequency were recovered.

(2) The true wild types grew normally on minimal medium and possessed glutamic dehydrogenase activity fully comparable to stock wild types. The frequencies of these wild types from the various intercrosses are not readily interpretable in terms of crossing-over within a linear arrangement of "sites", and it is suggested that at least some of the wild types may be due to gene conversion (transmutation).

(3) The growth of pseudowilds on minimal medium was intermediate between that usual for am and stock wild-type strains, and they possessed low glutamic dehydrogenase activity.

(4) am strains with no detectable glutamic dehydrogenase activity were isolated from pseudowilds and heterocaryons were reconstituted from them. These heterocaryons were shown to possess low enzyme activity similar to the pseudowilds. Thus certain am heteroalleles, which individually in homocaryons are incapable of producing detectable glutamic dehydrogenase activity, are complementary in function when present in different nuclei in a heterocaryon, with the resultant production of enzyme.

(5) Forced heterocaryons were made between the various am mutants in all possible combinations. Pairs of heteroalleles which 
had given pseudowilds in sexual crosses formed glutamic dehydrogenase in heterocaryons; pairs of heteroalleles which had given no pseudowilds in sexual crosses formed no glutamic dehydrogenase in heterocaryons.

(6) Homocaryotic $a m^{l}$ strains produce a qualitatively different glutamic dehydrogenase from the wild type, but $a m^{l}$ is complementary with certain other am heteroalleles, since forced heterocaryons produce greater or smaller amounts of apparently normal glutamic dehydrogenase in addition to the $a m^{l}$ enzyme.

\section{REFERENCES}

BEADLE, G. W. I957. The role of the nucleus in heredity. Symposium on The Chemical Basis of Heredity. Johns Hopkins Press.

BENZER, s. I957. The elementary units of heredity. Symposium on The Chemical Basis of Heredity. Johns Hopkins Press.

CAtcheside, D. G. 1956. Genes-their nature and function. Compt.-rend. Lab. Carlsberg, Ser. physiol., 26, 3 I-39.

FINCHAM, J. R. S. I954. Effects of a gene mutation in Neurospora crassa relating to glutamic dehydrogenase formation. 7. gen. Microbiol., ${ }_{11}, 236-246$.

FINCHAM, J. R. S. I957. A modified glutamic acid dehydrogenase as a result of gene mutation in Neurospora crassa. Biochem. 7., 65, 72 I-8.

FINCHAM, J. R. S., AND PATEMAN, J. A. I957a. Formation of an enzyme through complementary action of mutant alleles in separate nuclei in a heterocaryon. Nature, $179,744^{1-742}$.

Fincham, J. R. S., And PAteman, J. A. 1957b. A new allele at the am locus of Neurospora crassa. 7. Genet., 55, 456-466.

GILES, N. H. I95I. Studies on the mechanism of reversion in biochemical mutants of Neurospora crassa. Cold Spring Harbor Symposia Quant. Biol., 16, 283-313.

GIles, N. H., PARTRIDGE, C. W. H., AND Nelson, N. J. 1957. The genetic control of adenylosuccinase in Neurospora crassa. P.N.A.S., 43, 305-31 7 .

LINDEGREN, C. C. I955. Non-Mendelian segregation in a single tetrad of Saccharomyces ascribed to gene conversion. Science, $121,605-607$.

MITCHELL, м. в. I $955 a$. Aberrant recombination of pyridoxine mutants of Neurospora. P.N.A.S., 4I, 2 I 5-220.

MITCHELl, м. в. I955 $b$. Further evidence of aberrant recombination in Neurospora. P.N.A.S., 4 I, 935-937.

NEWMEYER, D. I 957 . Arginine synthesis in Neurospora crassa: genetic studies. 7. gen. Microbiol., ${ }_{1} 6,449-462$.

pateman, J. A. 1956. Mutation and adaptation studies in Neurospora. Ph.D. Thesis, University of London.

Pateman, J. A. I957. Backmutation studies at the am locus in Neurospora crassa. 7. Genet., 55, 444-445.

PitTENGer, T. H. I954. The general incidence of pseudo-wild types in Nearospora crassa. Genetics, 39, 326-342.

PONTECORVO, G., ROPER, J. A., HemMons, L. M., MACDONALD, K. D., AND BUfTON, A. W. J. I953. The genetics of Aspergillus nidulans. Adv. Genet., 5, I4 I-238.

PRITCHARD, R. H. I 955. The linear arrangement of a series of alleles of Aspergillus nidulans. Heredity, 9, 343-371.

Roman, H. 1956. Studies of gene mutation in Saccharomyces. Cold Spring Harbor Symposia Quant. Biol., 2I, 1 75-183.

SPIEGELMAN, s. I956. On the nature of the enzyme-forming system (with added conment by S. F. Velick). Enzymes: Units of Structure and Function (Gaebler, O. H., ed.), Academic Press, New York. 
ST LAWRENCE, P. 1956. The $q$ locus of Neurospora crassa. P.N.A.S., 42, 189 . ST LAWRENCE, P., AND BONNER, D. M. 1957. Gene conversions and problems of allelism. Symposium on The Chemical Basis of Heredity. Johns Hopkins Press. VOGEL, H. J., AND BONNER, D. M. 1956. A convenient growth medium for Neurospora (Medium N). Microbial Genetics Bulletin, No. I3.

WestergaARD, M., AND MItchell, H. K. 1947. Neurospora V. A synthetic medium favouring sexual reproduction. Amer. $\mathcal{F}$. Bot., 34, 573-578.

WINGE, ø. 1955. On interallelic crossing over. Heredity, 9, 373-384. 